\title{
Oligometastatic non-small cell lung cancer: from biology to clinical practice
}

Successful treatment of any solid malignancy at any stage results from the eradication (or long-term control) of all gross disease and of micrometastatic deposits.

In stage IV cancer, only systemic treatment may be able to control gross and invisible metastatic deposits scattered in distant organs and hopefully keep in check what is in fact a systemic disease. Local therapy is considered ineffective and only applied for temporarily palliating local symptoms on the ground that metastatic deposits will continuously reappear and progress leading to the patient's death even when all gross disease can be extirpated. Standard treatment of stage IV non-small cell lung cancer (NSCLC) is based on this tenet.

In 1995, Hellman and Weichselbaum (1) postulated the existence of a special category of stage IV cancer, with a reduced tendency to widespread dissemination, the hallmark of which was the appearance of a limited number of metastatic deposits (hence it was called oligometastatic).

In such cases eradication of all gross metastatic lesions could be proposed on the ground that diffuse micrometastatic seeding in the rest of the body had not yet occurred or that micrometastases were being kept in check. As a matter of fact, colorectal cancer liver metastases and pulmonary metastasis from sarcoma were already surgically treated at that time in an attempt to provide cure or prolong survival.

Eventually, the concept of oligometastatic disease (OMD) was also applied to NSCLC patients. Patients with a single brain or adrenal metastasis are routinely offered surgery or stereotactic body radiation therapy (SBRT) both to the primary and to the secondary lesion; and encouraging results of local radical treatment of up to 5 metastatic sites have been reported in several retrospective series.

In this series, several crucial aspects of oligometastatic NSCLC lung cancer (OM-NSCLC) will be covered, all pertinent to the management of OMD. We believe that some of this knowledge may become useful in the future for the management of stage IV lung cancer in general.

\section{How can we predict the biological behavior of stage IV lung cancer and identify OMD?}

The current gold standard for diagnosing OMD relies on counting the number of metastases or metastatic sites and determine whether they are susceptible to local treatment, with the aim to prolong survival. The current consensus definition of OMD NSCLC has operational value in that it provides a common language base for everybody, helps design and compare data from future investigations, but it has some limitations. Since it includes the feasibility of local therapy to all disease sites in order to improve outcomes, it contains a somewhat circular reference: local therapy can be recommended for a patient with OMD to improve outcomes, and the patient has OMD if local therapy is feasible and can be proposed to improve outcomes (2).

Most importantly, it does not (cannot) account for tumor markers, genomic signatures and microenvironmental factors that have a strong influence on the clinical course of the disease and could define this subclass of stage IV patients on biological grounds. The general concept of biology versus consensus definition is introduced by Mentink, Dingemans and colleagues.

One of the main and so far, unresolved problem is the identification of true OMD as opposed to a transient brief phase in which few metastatic lesions are detected just before widespread dissemination occurs. Is OMD truly the result of metastatic clones appearing later during the course of the disease, giving us a window of opportunity for local ablative therapy (LAT) to eradicate the cell factory that will eventually give rise to polymetastatic incurable cancer? The recognition of such biological profile would enable us to select stage IV patients appropriately for local therapy alone, or in combination with systemic treatments, or for systemic treatments only.

Spectacular advances in the understanding of cancer biology and of the metastatic process are being made at a steady pace. Rashdan, Gerber and colleagues have analyzed up-to-date available knowledge about biological markers and potential regulators of the metastatic cascade and their interactions with the microenvironment that may lead to a downregulation of the metastatic process, while Belluomini, Milella and colleagues have extensively reviewed the metastatic process with special 
attention to the role of microenvironmental factors.

\section{How frequent is OMD?}

With the widespread adoption of sensitive imaging technology such as ${ }^{18}$ fluorodeoxyglucose-positron emission tomography $\left({ }^{18} \mathrm{~F}\right.$-FDG-PET) in daily practice, patients with clinically unsuspected metastatic lesions are now frequently identified, but the actual frequency of OMD is yet to be clarified as data in the literature are of poor quality (in that they lack a denominator) and to some extent contradictory. However, such information would be extremely important for the design of appropriately sized trials and for resource allocation in daily clinical practice. Gobbini, Giaj-Levra and colleagues have extensively reviewed all available information to try to come up with a sound answer.

\section{Which is the best management algorithm for wild-type OM-NSCLC? How to integrate local treatment with immune-checkpoint inhibitors or tyrosine-kinase inhibitors?}

Cortinovis, Rolfo and colleagues have explored and extensively reviewed the potential of liquid biopsy to diagnose, monitor and predict the effects of treatment by searching for blood-based biomarkers, thus obviating the need to repeat invasive tissue biopsies and facilitating the identification of potentially effective new treatment lines during the course of the disease rather than using stored tissue samples from the primary lesion, thus allowing us to adapt our therapeutic schemes as the clinical picture evolves with the appearance of resistance to systemic agents.

Fallet, Cadranel and colleagues provide us with a beautiful systematic review on the potential benefits of local treatment in patients with driver mutations, while Hendriks and colleagues address methodological questions and challenges of combining Immune checkpoint Inhibitors with LAT/LCT.

\section{Which sequence for systemic and local therapies is more appropriate?}

By LAT, it is understood that the tumor deposits have been eradicated by stereotactic ablative radiotherapy (SABR) or surgery. This may have occurred either upfront (before any systemic treatment has been given) or subsequently, whereas by local consolidative therapy (LCT), it is understood that systemic treatment has been given upfront and then the tumor deposits have been treated by SABR or surgery, if and only if widespread dissemination has not occurred in the mean time.

The best timing for administering local treatment in OM-NSCLC is unclear and highly debated: is upfront local therapy as effective and safe as local therapy administered after induction systemic treatment with no sign of progression? Although it may be reasonable to wait for the clinical behavior of OMD to declare itself before administering local treatment, on the other side upfront administration of local therapy might possibly prevent more aggressive subclones from originating in anyone of the detectable disease sites. This debated topic is covered by Tjong, Siva and colleagues.

\section{Which is the best form of local treatment? What are than the best means to achieve local control of oligometastatic deposits?}

Several options are available and each one of them is examined in this issue in detail. No local treatment is absolutely safe, and all of them are costly and can cause considerable discomfort.

Sundahl, Lievens and colleagues cover the best ways to administer SABR the efficacy of which depends on radiation dose constraints and organs involved, and synergize it with systemic treatments.

Surgical removal of metastatic deposits is more invasive than SABR, although recent minimally invasive approaches have made it more tolerable and reduced side effects to a great extent. Surgical resection also offers an opportunity to stage the mediastinum and collect large tissue samples to study the biology of metastasis and their interactions with the microenvironment in which they grow. Berzenji, Van Schil and colleagues cover this topic while prognostic factors associated with best outcomes after surgical LAT/LCT are examined by Bertolaccini, Spaggiari and colleagues. 
The potential utility of endo-bronchial ablation techniques is nicely reviewed by Olive, Fong and colleagues who provide us with a very clear and updated review of current technological developments.

\section{Does a prolonged disease-free interval after LAT/LCT reflect a slower pace of tumor progression or is it the effect of local therapy?}

Local ablative/consolidative treatment to patients with OM-NSCLC has been widely accepted based on observational data and recently on small phase 2 randomized trials. Unfortunately, when you deal with small numbers false positive results cannot be ruled out (3). It is recognized therefore that randomized phase 3 trials are still necessary and their results should be awaited to establish such a new treatment strategy on solid grounds. Current efforts have been summarized for the readers by Brandao, Berghmans and colleagues with an updated review of ongoing trials of local ablative/consolidative therapy in the OM-NSCLC setting, and finally discussed by Bungaro, Novello and colleagues.

\section{Acknowledgments}

We first of all would like to thank all authors and co-authors for their outstanding contributions to this special series. We would also like to thank the Editorial Office of TLCR for their assistance and the Editor of the Journal for giving us the opportunity to publish this special series on Oligometastatic Non-small Cell Lung Cancer. We hope it will help the readers of TLCR to gain sound and updated knowledge on this topic and that it will stimulate further research efforts in this exciting multidisciplinary area of clinical oncology.

Funding: None.

\section{Footnote}

Provenance and Peer Review: This article was commissioned by the editorial office, Translational Lung Cancer Research for the series "Oligometastatic NSCLC: definition and treatment opportunities". The article did not undergo external peer review.

Conflicts of Interest: Both authors have completed the ICMJE uniform disclosure form (available at https://dx.doi. org/10.21037/tlcr-21-533). The series "Oligometastatic NSCLC: definition and treatment opportunities" was commissioned by the editorial office without any funding or sponsorship. MVI and TB served as the unpaid Guest Editors of the series and MVI serves as an unpaid editorial board member of Translational Lung Cancer Research from Oct 2019 to Oct 2021. TB receives consulting fees from Inhatarget, payment from Bayer and participation fees for advisory board from Roche, Bayer and Janssen. The authors have no other conflicts of interest to declare.

Ethical Statement: The authors are accountable for all aspects of the work in ensuring that questions related to the accuracy or integrity of any part of the work are appropriately investigated and resolved.

Open Access Statement: This is an Open Access article distributed in accordance with the Creative Commons AttributionNonCommercial-NoDerivs 4.0 International License (CC BY-NC-ND 4.0), which permits the non-commercial replication and distribution of the article with the strict proviso that no changes or edits are made and the original work is properly cited (including links to both the formal publication through the relevant DOI and the license). See: https:// creativecommons.org/licenses/by-nc-nd/4.0/.

\section{References}

1. Hellman S, Weichselbaum RR. Oligometastases. J Clin Oncol 1995;13:8-10.

2. Dingemans AC, Hendriks LEL, Berghmans T, et al. Definition of Synchronous Oligometastatic Non-Small Cell Lung 
Cancer-A Consensus Report. J Thorac Oncol 2019;14:2109-19.

3. Lara PN, Redman MW. The hazards of randomized phase II trials. Ann Oncol 2012;23:7-9. Erratum in: Ann Oncol. 2012 Mar;23(3):810-2.

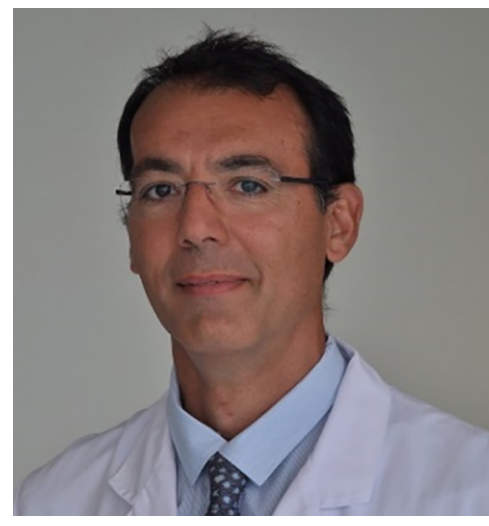

Maurizio V. Infante

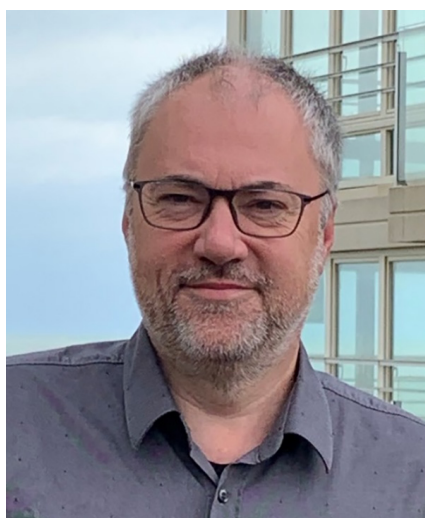

Thierry Berghmans

Maurizio V. Infante, MD Head of Thoracic Surgery Department, University and Hospital Trust, Verona, Italy. (Email: Maurizio.Infante@aovr.veneto.it) Thierry Berghmans, MD, PhD Department of Intensive Care and Oncologic Emergencies \& Thoracic Oncology, Institute Fules Bordet, Université Libre de Bruxelles, Brussels, Belgium. (Email: thierry.berghmans@bordet.be) Submitted Jul 01, 2021. Accepted for publication Jul 17, 2021. doi: $10.21037 /$ tlcr-21-533

View tis article at: https://dx.doi.org/10.21037/tlcr-21-533

Cite this article as: Infante MV, Berghmans T. Oligometastatic non-small cell lung cancer: from biology to clinical practice. Transl Lung Cancer Res 2021;10(7):3320-3323. doi: 10.21037/ tlcr-21-533 